Submitted to ApJ Letters 27 August 2012; AcCepted 14 November 2012

Preprint typeset using $\mathrm{IAT}_{\mathrm{E}} \mathrm{X}$ style emulateapj v. 5/2/11

\title{
DETECTION OF RADIO EMISSION FROM THE HYPERACTIVE L DWARF 2MASS J13153094-2649513AB
}

\author{
Adam J. Burgasser ${ }^{1}$, CArl Melis ${ }^{1,2}$, B. Ashley Zauderer ${ }^{3}$, And Edo Berger ${ }^{3}$ \\ Submitted to ApJ Letters 27 August 2012; Accepted 14 November 2012
}

\begin{abstract}
We report the detection of radio emission from the unusually active L5e + T7 binary 2MASS J13153094-2649513AB made with the Australian Telescope Compact Array. Observations at 5.5 GHz reveal an unresolved source with a continuum flux of $370 \pm 50 \mu \mathrm{Jy}$, corresponding to a radio luminosity of $L_{r a d}=\nu L_{\nu}=(9 \pm 3) \times 10^{23} \mathrm{erg} \mathrm{s}^{-1}$ and $\log _{10} L_{r a d} / L_{b o l}=-5.44 \pm 0.22$. No detection is made at $9.0 \mathrm{GHz}$ to a $5 \sigma$ limit of $290 \mu \mathrm{Jy}$, consistent with a power law spectrum $S_{\nu} \propto \nu^{-\alpha}$ with $\alpha \gtrsim 0.5$. The emission is quiescent, with no evidence of variability or bursts over $3 \mathrm{hr}$ of observation, and no measurable polarization ( $\mathrm{V} / \mathrm{I}<34 \%$ ). 2MASS J1315-2649AB is one of the most radio-luminous ultracool dwarfs detected in quiescent emission to date, comparable in strength to other cool sources detected in outburst. Its detection indicates no decline in radio flux through the mid-L dwarfs. It is unique among $\mathrm{L}$ dwarfs in having strong and persistent $\mathrm{H} \alpha$ and radio emission, indicating the coexistence of a cool, neutral photosphere (low electron density) and a highly active chromosphere (high electron density and active heating). These traits, coupled with the system's mature age and substellar secondary, makes 2MASS J1315-2649AB an important test for proposed radio emission mechanisms in ultracool dwarfs.
\end{abstract}

Subject headings: stars: chromospheres — stars: individual (2MASS J13153094-2649513) — stars: brown dwarfs - stars: low mass — stars: magnetic field

\section{INTRODUCTION}

The origin of radio emission from ultracool dwarfs (UCDs; late M-, L- and T-type stars and brown dwarfs with $\left.\mathrm{T}_{\text {eff }} \lesssim 3000 \mathrm{~K}\right)$ remains one of the great mysteries in our understanding of these cool, lowmass objects. The first radio detection of the M9 brown dwarf LP 944-20 (Berger et al. 2001) was unexpected given the precipitous decline in the strength and incidence of optical and X-ray emission among late-M and $\mathrm{L}$ dwarfs $\left(\log L_{H \alpha} / L_{b o l} \approx \log L_{X} / L_{b o l} \approx\right.$ -4 to $\lesssim-6$; Gizis et al. 2000; Stelzer et al. 2006; Schmidt et al. 2007) and the correlation between X-ray and radio flux among many stellar sources (i.e., the Guedel-Benz relation, $\log _{10} L_{\nu, \text { rad }} / L_{X} \approx-15.5 \mathrm{~Hz}^{-1}$; Guedel \& Benz 1993; Benz \& Guedel 1994). In the chromospheric evaporation model of solar and stellar flares (Machado et al. 1980; Allred et al. 2006), the radio/Xray correlation is attributed to the heating and evaporation of chromospheric plasmas (X-ray emission) by accelerated electrons (radio emission) via the Neupert effect (Neupert 1968). The dozen UCDs detected in the radio to date (Berger 2002, 2006; Burgasser \& Putman 2005; Phan-Bao et al. 2007; Antonova et al. 2008; McLean et al. 2011, 2012; Route \& Wolszczan 2012) violate the Guedel-Benz relation by orders of magnitude, suggesting a breakdown in this mechanism. UCD radio emission also exhibits a broad range of behaviors, including low-polarization quiescent emission (e.g., Berger 2002), sporadic variability (e.g., Antonova et al. 2007), periodic variability synched with

\footnotetext{
${ }^{1}$ Center for Astrophysics and Space Science, University of California San Diego, La Jolla, CA 92093, USA; aburgasser@ucsd.edu

2 Joint NSF AAPF Fellow and CASS Postdoctoral Fellow

${ }^{3}$ Harvard-Smithsonian Center for Astrophysics, 60 Garden Street, Cambridge, MA 02138, USA
}

rotation (e.g., Berger et al. 2005; McLean et al. 2011), highly polarized bursts (e.g., Burgasser \& Putman 2005), and "pulsar-like" rotationally-synched coherent emission (e.g., Hallinan et al. 2007; Berger et al. 2009). The complexity of these behaviors, and the decoupling of radio, X-ray and $\mathrm{H} \alpha$ emission trends, has stimulated new theoretical work on the origin of magnetic emission in very cool stars, brown dwarfs and exoplanets (e.g., Schrijver 2009; Reiners \& Christensen 2010; Kuznetsov et al. 2012).

While the decline in optical emission in UCDs is a general trend, a small number of unusually "hyperactive" cool dwarfs have been identified, whose strong and persistent $\mathrm{H} \alpha$ emission also remains a mystery (Burgasser et al. 2000; Liebert et al. 2003; Schmidt et al. 2007). One such source is the L5e 2MASS J13153094-2649513 (hereafter 2MASS J1315-2649; Hall 2002; Gizis 2002), a high proper motion, very cool dwarf that has exhibited pronounced and sustained $\mathrm{H} \alpha$ emission at the level of $\log _{10} L_{H \alpha} / L_{b o l} \approx-4$ for over a decade (Hall 2002; Gizis 2002; Fuhrmeister et al. 2005; Burgasser et al. 2011). The optical emission, which includes Ca II and alkali resonance lines, is $\sim 100$ times stronger than equivalently classified L dwarfs. 2MASS J1315-2649 also harbors a resolved $\mathrm{T}$ dwarf companion, which appears to be too widely separated to induce magnetic interaction (Burgasser et al. 2011). Kinematics and coevality analyses indicate that this is a mature system (few Gyr) and that 2MASS J1315-2649A is likely a low mass star just above the hydrogen burning mass limit $\left(\mathrm{M} \sim 0.075 \mathrm{M}_{\odot}\right)$. Its strong optical emission therefore contradicts stellar age-activity trends (Fleming et al. 1995), again pointing to new magnetic behavior in the UCD regime.

In this Letter, we report the detection of quiescent, unpolarized radio emission from 2MASS J1315-2649 at 
5.5 GHz based on observations from the Australian Telescope Compact Array (ATCA). The radio luminosity of this source exceeds all other UCDs detected in quiescent emission to date, but is consistent with a lower radio to $\mathrm{H} \alpha$ luminosity fraction than other L dwarfs. In Section 2 we summarize the observations, data analysis and resulting measurements. In Section 3 we use these data to infer the properties of the emitting region and compare to other UCD emitters. In Section 4 we discuss the possible origins of this emission and propose future observations.

\section{OBSERVATIONS}

2MASS J1315-2649 was observed with ATCA in the compact hybrid H214 configuration (baselines of 0.082 $5.94 \mathrm{~km}$ ) on 14 July 2011 (UT). Continuum observations were taken in dual-sideband mode simultaneously at 5.5 $\mathrm{GHz}$ (C-band) and 9.0 GHz (X-band). The Compact Array Broadband Backend (CABB; Wilson et al. 2011) was used, providing $2 \mathrm{GHz}$ bandwidth per observing frequency in 2048 channels of $1 \mathrm{MHz}$ width each. The source was tracked for a total of $3 \mathrm{hr}$ in $5 \mathrm{~min}$ and $10 \mathrm{~min}$ intervals, interspersed with observations of the gain calibrator QSO B1255-316. The quasar QSO B1934-638 was used for primary flux calibration, while QSO B0823-500 was used for bandpass calibration. Data were reduced using the Astronomical Image Processing System package (AIPS; Greisen 2003) following best practices for wideband data reduction.

Images of the 2MASS J1315-2649 field from the integrated broadband $5.5 \mathrm{GHz}$ and $9.0 \mathrm{GHz}$ data are shown in Figure 1, with synthesized beam sizes $\left(B_{\nu}\right)$ of $\approx 30^{\prime \prime}$ and $\approx 25^{\prime \prime}$, respectively. Several significant sources are seen in each field, but in the $5.5 \mathrm{GHz}$ data there is one source centered att $\left(13^{h} 15^{m} 29.99\right.$, $\left.-26^{\circ} 49^{\prime} 55^{\prime \prime} .7\right) \pm\left(0.12,2^{\prime \prime}\right.$. 0$)$ that is coincident with the proper-motion-corrected position of 2MASS J1315-2649 to within $2 \sigma$ 专 This source has an integrated flux density of $S_{5.5}=370 \pm 50 \mu \mathrm{Jy}$, a $7.4 \sigma$ detection. We rule out source confusion as the origin of this emission by noting that the deep $5 \mathrm{GHz}$ survey of the Lockman Hole by Ciliegi et al. (2003) measured a source density of $N$ $=0.04 \mathrm{arcmin}^{-2}$ for $S_{5.0}>350 \mu \mathrm{Jy}$, implying a confusion probability $1-e^{-N B_{5.5}^{2}} \approx 1 \%$ for this source. No equivalent source is detected in the $9.0 \mathrm{GHz}$ data to a $5 \sigma$ limiting flux of $290 \mu \mathrm{Jy}$.

The 5.5 GHz emission from 2MASS J1315-2649 appears to be roughly constant over the observing period. Dividing the data into two equally-spaced periods yields consistent fluxes of $430 \pm 60 \mu \mathrm{Jy}$ and $340 \pm 70 \mu \mathrm{Jy}$. Further division fails to yield a significant detection on timescales of $10 \mathrm{~s}$ to $1 \mathrm{hr}$, indicating a bursting flux limit of $<0.5 \mathrm{mJy}(<1.3 \mathrm{mJy})$ for a $30 \mathrm{~min}(5 \mathrm{~min})$ event, timescales typical of previously observed radio bursts (e.g., Berger 2002; Burgasser \& Putman 2005; Hallinan et al. 2007; Berger et al. 2009). There is a suggestion of spectral structure in the emission, as division of the time-integrated flux is slightly dominated by the

\footnotetext{
${ }^{4}$ Equinox J2000 coordinates

5 The source located 5!4 northeast of 2MASS J1315-2649 is NVSS J131549-264647 (Condon et al. 1998; $S_{1.4}=13.8 \pm 0.6 \mathrm{mJy}$, $S_{5.5} \approx 3 \mathrm{mJy}$ ). Its dirty beam pattern was cleaned using standard AIPS routines, and does not contribute to the flux detected at the position of 2MASS J1315-2649.
}

TABLE 1

Radio Properties of 2MASS J13153094-2649513

\begin{tabular}{|c|c|}
\hline Parameter & Value \\
\hline $\begin{array}{l}\text { Right Ascension } \\
\text { Declination }^{\mathrm{a}} \\
S_{5.5}(\mu \mathrm{Jy}) \\
S_{9.0}(\mu \mathrm{Jy}) \\
\alpha^{\mathrm{c}} \\
\log _{10} L_{5.5}\left(\mathrm{erg} \mathrm{s}^{-1} \mathrm{~Hz}^{-1}\right) \\
\log _{10} \nu L_{\nu}\left(\mathrm{erg} \mathrm{s}^{-1}\right) \\
\log _{10} \nu L_{\nu} / L_{b o l} \\
\log _{10} \nu L_{\nu} / L_{H \alpha}\end{array}$ & $\begin{array}{c}13^{h} 15^{m} 29^{\mathrm{s}} .99 \pm 0.12 \\
-26^{\circ} 49^{\prime} 55^{\prime \prime} 7 \pm 2^{\prime \prime} 0 \\
370 \pm 50 \\
<290^{\mathrm{b}} \\
\gtrsim 0.5 \\
14.18 \pm 0.15 \\
23.92 \pm 0.15 \\
-5.44 \pm 0.22 \\
-1.3 \pm 0.5^{\mathrm{d}}\end{array}$ \\
\hline
\end{tabular}

${ }^{a}$ Equinox J2000 coordinates on Julian data 2455757.

b $5 \sigma$ upper limit.

c Assuming $S_{\nu} \propto \nu^{-\alpha}$.

d Accounting for \pm 0.4 dex variation in $\mathrm{H} \alpha \mathrm{EW}$ measurements reported in the literature.

lowest frequency channels $(\lesssim 5.2 \mathrm{GHz})$, consistent with a negative power-law spectral slope (see below). However, these differences are not statistically significant. We also detect no significant polarization in the emission, with a Stokes $V / I 3 \sigma$ limit of $\lesssim 34 \%$.

\section{CHARACTERIZING THE RADIO EMISSION OF}

The magnitude of the radio emission from 2MASS J1315-2649 is remarkable, particularly given the late spectral types of its components. For the following, we assume the emission arises from the L5 primary (see Section 4 for discussion on possible contribution from the secondary). The absolute radio intensity at $5.5 \mathrm{GHz}$ is $L_{\nu, \mathrm{rad}}=4 \pi d^{2} S_{5.5}=$ $(1.6 \pm 0.6) \times 10^{14} \mathrm{erg} \mathrm{s}^{-1} \mathrm{~Hz}^{-1}$ based on the estimated $d$ $=19 \pm 3 \mathrm{pc}$ distance of the 2MASS J1315-2649 system. Parameterizing the radio power as $L_{r a d}=\nu L_{\nu}$, we find6 $L_{r a d}=(9 \pm 3) \times 10^{23} \mathrm{erg} \mathrm{s}^{-1}$, corresponding to $\log _{10} L_{\text {rad }} / L_{\text {bol }}=-5.44 \pm 0.22$ assuming a bolometric luminosity of $\log _{10} L_{b o l} / L_{\odot}=-4.19 \pm 0.16$ for the primary (Burgasser et al. 2011). The lack of emission at $9.0 \mathrm{GHz}$ implies a power-law slope in the radio flux $S_{\nu} \propto \nu^{-\alpha}$ with $\alpha \gtrsim 0.5$. This index is broadly consistent with the radio spectra of other UCDs (Osten et al. 2009; Ravi et al. 2011), with the exception of the flat-spectrum radio source 2MASS J1314+1320 (McLean et al. 2011).

To place this emission in context, we compare the radio luminosity of 2MASS J1315-2649 to other UCDs in Figure 2. We find 2MASS J1315-2649 to be the most radio-luminous quiescent emitter among this group, comparable to the peak fluxes detected among UCD radio outbursts. Its brightness is consistent with no appreciable decline in radio power with spectral type well into the L dwarf class, at least for radio-loud UCDs (Audard et al. 2007; Berger et al. 2010). No X-ray measurement has yet been made of 2MASS J1315-2649, but we can infer that it likely violates the Guedel-Benz relation since it would require an X-ray luminosity $\log _{10} L_{X}$

${ }^{6}$ Alternately, adopting a spectral flux distribution that peaks around $5.5 \mathrm{GHz}$, with $S_{\nu} \propto \nu^{2.5}$ for $\nu<=5.5 \mathrm{GHz}$ and $S_{\nu} \propto \nu^{-1.5}$ for $\nu>5.5 \mathrm{GHz}$ (Dulk 1985), and integrating over 6 decibels about $5.5 \mathrm{GHz}$, we derive a statistically equivalent $L_{\text {rad }}$ $=(1.5 \pm 0.5) \times 10^{24} \mathrm{erg} \mathrm{s}^{-1}$. 

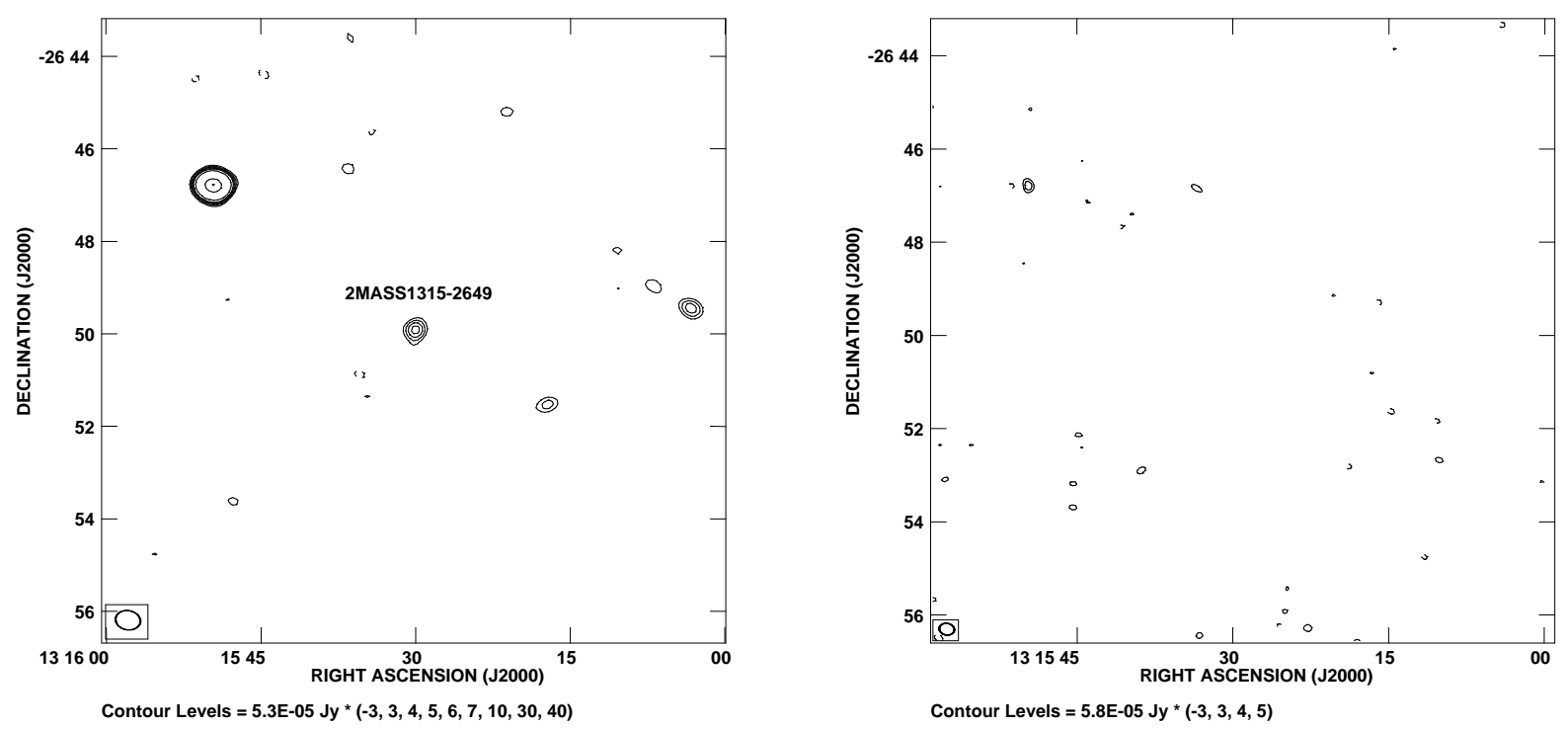

FIG. 1. - $13^{\prime} \times 13^{\prime}$ contour images of the 2MASS J1315-2649 field in integrated $5.5 \mathrm{GHz}$ (left) and $9.0 \mathrm{GHz}$ (right) flux density, based on our ATCA observations. Contours are spaced at listed intervals of the noise $\left(53 \mu \mathrm{Jy} \mathrm{beam}^{-1}\right.$ in the $5.5 \mathrm{GHz}^{\mathrm{Hata}}, 58 \mu \mathrm{Jy}$ beam ${ }^{-1}$ in the 9.0 GHz data; negative contours are dashed) and the beam shape is indicated in the lower left corners. 2MASS J1315-2649 is detected at $5.5 \mathrm{GHz}$ with $7.4 \sigma$ confidence.
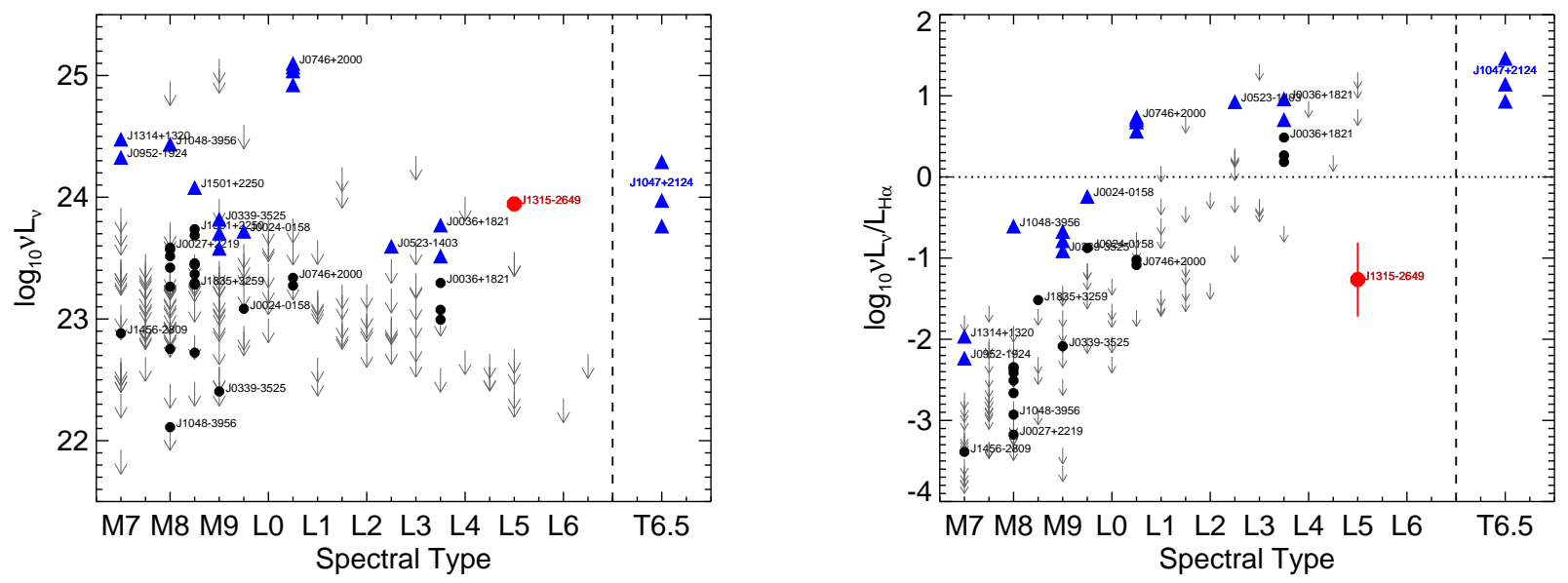

FIG. 2.- (Left): Radio luminosity $\left(\nu L_{\nu}\right)$ versus spectral type for M7 and later sources, based on the compilation of McLean et al. (2012) supplemented with results from Osten et al. (2006, 2009); Route \& Wolszczan (2012); and this study. Detections in quiescent (black circles) and peak outburst (blue triangles) are labelled by source sexigessimal coordinates; upper limits are indicated by downward arrows. Quiescent emission from 2MASS J1315-2649, with $\log \nu L_{\nu}=23.92 \pm 0.15$ at $5.5 \mathrm{GHz}$, is indicated by the red circle. Spectral type uncertainties are typically \pm 0.5 subtypes. (Right): Ratio of radio to $\mathrm{H} \alpha$ luminosity as a function of spectral type (same symbols). Only sources with $\mathrm{H} \alpha$ detections are plotted. The relative emission of 2MASS J1315-2649, $\log \nu L_{\nu} / L_{H \alpha}=-1.3 \pm 0.4$ (uncertinaty includes scatter in the observed $\mathrm{H} \alpha$ measurements), is distinct from the other $\mathrm{L}$ dwarfs and more consistent with a late $\mathrm{M}$ dwarf.

$\approx 29.7 \mathrm{erg} \mathrm{s}^{-1}$, or $\log _{10} L_{X} / L_{\text {bol }}>0$ (this is also true for the secondary). Active $\mathrm{M}$ and $\mathrm{L}$ dwarfs typically have $\log _{10} L_{X} / L_{H \alpha} \approx 0-1$ (Berger et al. 2010), so even with its strong optical emission 2MASS J1315-2649 likely has $\log _{10} L_{X} / L_{b o l} \approx-3.5$ and $\log _{10} L_{\nu, \mathrm{rad}} / L_{X} \approx$ $-11.8 \mathrm{~Hz}^{-1}$, violating the Guedel-Benz relation by nearly four orders of magnitude.

Figure 2 also displays the (asynchronous) ratio of radio and $\mathrm{H} \alpha$ luminosities for UCDs observed to date. This ratio steadily increases from late-M to mid-L types, in both quiescent and flaring fluxes, reflecting the decline in optical emission and plateau in radio emission with later spectral type. Nearly all L and T dwarfs appear to emit more nonthermal power in the radio than in optical lines.
2MASS J1315-2649 is a obvious exception; its strong $\mathrm{H} \alpha$ emission implies $\log _{10} \nu L_{\nu} / L_{H \alpha}=-1.3 \pm 0.5$ (taking into account scatter in the $\mathrm{H} \alpha$ measurements), a ratio more in line with mid- and late-M dwarfs. Given that no latertype source has yet been detected in quiescent flux (the T6.5 2MASS J1047+2124 has only been detected in outburst; Route \& Wolszczan 2012), we can only speculate as to whether this measurement reflects a change in the nonthermal emission spectrum of UCDs at lower temperatures or the unique nature of 2MASS J1315-2649 itself.

\section{ORIGINS FOR THE EMISSION}


The lack of measurable polarization or variability during our $3 \mathrm{hr}$ observation of 2MASS J1315-2649 suggests that its radio emission may be driven by incoherent gyrosychrotron emission (Dulk 1985), as has been previously proposed for quiescent UCD radio sources (Berger 2002; Osten et al. 2006; Ravi et al. 2011). This emission peaks at high harmonics of the electron cyclotron frequency, $\nu_{\text {peak }}=s \nu_{c}=2.8 s B \mathrm{MHz}$, where $s=10$ $100, B$ is local magnetic field strength in Gauss, and $\nu_{c}=e B / 2 \pi m_{e} c$ is the electron cyclotron frequency. Assuming $\nu_{\text {peak }} \lesssim 5 \mathrm{GHz}$ implies $B \lesssim 20-200 \mathrm{G}$, on par with prior estimates for UCD radio emission regions (Berger 2006). Razin-Tsytovich suppression also provides a constraint on the electron number density, requiring $\nu_{\text {peak }}>\nu_{p}^{2} / \nu_{c} \approx 29 n_{e} / B \mathrm{~Hz}$, or $n_{e} \lesssim 10^{9}-10^{10} \mathrm{~cm}^{-3}$, where $\nu_{p}=\left(n_{e} e^{2} / \pi m_{e}\right)^{1 / 2}$ is the electron plasma frequency. The emitting region scalelength $(L)$ is constrained by the brightness temperature of the emission, $T_{b r} \approx 10^{10}\left(L / \mathrm{R}_{J u p}\right)^{-2} \mathrm{~K}$, which cannot exceed the effective temperature of the emitting electrons $7, \mathrm{~T}_{\text {eff }}$ $\approx 10^{9}-10^{10} \mathrm{~K} \approx 0.1-1 \mathrm{MeV}$. This constraint yields $L \gtrsim 1-3 \mathrm{R}_{\text {Jup }}$, or $1-3$ stellar radii, a scale that is comparable to resolved radio sources associated with M dwarf coronae (Alef et al. 1997) and orders of magnitude smaller than the 2MASS J1315-2649AB separation $\left(\approx 10^{4}\right.$ radii $)$. This scale size is also equivalent to the corotation orbital radius 8 for a $0.08 \mathrm{M}_{\odot}$ UCD dwarf with a rotation period of $1.6 \mathrm{hr}$, suggesting that if 2MASS J1315-2649 is a rapid rotator (its rotation period is currently unknown), coronal stripping may play a role in the emission geometry (James et al. 2000; Berger et al. 2008; Ravi et al. 2011). Assuming the presence of a dipole field that scales as $B \propto R^{-3}$, these values suggest a surface field of roughly $1-5 \mathrm{kG}$, on par with Zeeman broadening measurements of late-M and L dwarf photospheres (Johns-Krull \& Valenti 1996; Reiners \& Basri 2010). Thus, gyrosynchrotron emission is a viable mechanism for the radio emission of 2MASS J1315-2649, provided that it has a sufficiently large magnetic field strength and structure.

Hallinan et al. (2007, 2008) have proposed an alternative mechanism for UCD radio emission, electron cyclotron masers (ECM; Wu \& Lee 1979) arising from plasma cavities near magnetic poles. ECM operates when $\nu_{c}>>\nu_{p}$, and thus favors environments with low electron densities and large field strengths like those of UCD atmospheres. Indeed, the increasingly neutral photospheres associated with declining photospheric temperatures has been proposed as an explanation for the decline in optical and X-ray emission (Mohanty et al. 2002). ECM provides a natural explanation for the highly-polarized, rotationally-modulated bursts observed in, e.g., TVLM 513-46546 and LSR J1835+3529, but Hallinan et al. (2008) also argue that unpolarized, broadband, quiescent emission may arise if ECM radiation is

\footnotetext{
7 This value assumes an electron energy distribution $N(E) \propto$ $E^{-\delta}$ with $\delta=2$ (consistent with $\left.S_{\nu} \propto \nu^{-0.5}\right), E>10 \mathrm{keV}, s=$ $10-100$, and that the emission is viewed perpendicular to the field; see Eqn. 37 of Dulk (1985)).

${ }^{8} R_{C} \equiv\left(G M P^{2} / 4 \pi^{2}\right)^{1 / 3} \approx 5(P / h r)^{2 / 3}\left(M / M_{\odot}\right)^{1 / 3} \mathrm{R}_{J u p}$ is the radius beyond which gravitational force cannot provide the necessary centripetal acceleration for solid body rotation with period $P$.

9 2MASS J1315-2649AB has a projected semi-major axis of 6.6 AU and estimated orbital period of $\sim 50 \mathrm{yr}$, implying a circular orbital velocity of $0.8 \mathrm{AU} \mathrm{yr}^{-1}$. For a relative astrometric precision of $100 \mu$ as, VLBI could in principle detect orbital motion on a daily basis.
}

dispersed in the ambient plasma or emitted from several discrete maser sources across the stellar surface. Dispersion may be particularly relevant in the magnetosphere of 2MASS J1315-2649 as its persistent and strong $\mathrm{H} \alpha$ emission indicates a heating source for coronal plasma; dispersion may have also enhanced its radio detectability if orientation effects are important (Hallinan et al. 2008). On the other hand, if plasma densities in the source region exceed $n_{e} \approx 2 \times 10^{3} B^{2} \approx 10^{9}-10^{10} \mathrm{~cm}^{-3}$ for a 1-3 kG field, both ECM and gyrosynchrotron emission may be suppressed (Zarka et al. 2001).

The calculations above have implicitly assumed that the emission arises from the L5 primary, but the resolution of ATCA cannot rule out emission from the closelyseparated T7 companion (338 mas; Burgasser et al. 2011), which has a similar spectral type as the T6.5 radio source 2MASS J10475385+2124234 (hereafter 2MASS J1047+2124; Route \& Wolszczan 2012). We assert that significant nonthermal emission from the secondary is unlikely, however, as this would require a very high, sustained radio luminosity $\left(\log _{10} L_{\text {rad }} / L_{b o l} \approx\right.$ -3.8 , on par with the peak emission during the rapid bursts from 2MASS J1047+2124), as well as an $\mathrm{H} \alpha$ luminosity that would exceed its bolometric luminosity $\left(\log _{10} L_{H \alpha} / L_{b o l} \approx+1.6\right)$. Nevertheless, resolved VLBI radio imaging of this binary could resolve the source(s) of emission, and potentially enable short-term astrometric orbit measurements to constrain both the component masses and the system age 9

The radio emission from 2MASS J1315-2649 may prove to be a critical test of the gyrosynchrotron and ECM models, given the strength of the quiescent emission, the low photospheric temperature (for either component), and evidence of a significant chromosphere. More extensive monitoring of this source is needed to assess whether and over what time scale the radio emission may be variable, which would constrain the length scale of the emission and hence $T_{b r}$; and also determine whether periodic bursting emission is present, direct evidence of a coherent emission process. Coincident monitoring of $\mathrm{H} \alpha$, radio and broadband emission would also probe the connection between photospheric and chromospheric structure; studies of structural connection for other UCDs have so far proven inconclusive (Berger et al. 2008, 2010). This oddball L dwarf may hold the key to understanding magnetic emission in UCDs in general.

The authors would like to thank Duty Astronomer Jay Blanchard for his assistance with the observations, and Juergen Ott for assistance in our application for ATCA green time. C.M. acknowledges support from the National Science Foundation under award No. AST1003318; E.B. acknowledges support from the National Science Foundation under award No. AST-1008361

Facilities: ATCA 


\section{REFERENCES}

Alef, W. Benz, A. O., \& Guedel, M. 1997, A\&A, 317, 707

Allred, J. C., Hawley, S. L., Abbett, W. P., \& Carlsson, M. 2006, ApJ, 644, 484

Antonova, A., Doyle, J. G., Hallinan, G., Bourke, S., \& Golden, A. 2008, A\&A, 487,317

Antonova, A., Doyle, J. G., Hallinan, G., Golden, A., \& Koen, C. 2007, A\&A, 472, 257

Audard, M., Osten, R. A., Brown, A., Briggs, K. R., Güdel, M., Hodges-Kluck, E., \& Gizis, J. E. 2007, A\&A, 471, L63

Benz, A. O., \& Guedel, M. 1994, A\&A, 285, 621

Berger, E. 2002, ApJ, 572, 503

—. 2006, ApJ, 648, 629

Berger, E., Ball, S., Becker, K. M., Clarke, M., Frail, D. A., Fukuda, T. A., Hoffman, I. M., Mellon, R., Momjian, E. Murphy, N. W., Teng, S. H., Woodruff, T., Zauderer, B. A., \& Zavala, R. T. 2001, Nature, 410, 338

Berger, E., Basri, G., Fleming, T. A., Giampapa, M. S., Gizis, J. E., Liebert, J., Martín, E., Phan-Bao, N., \& Rutledge, R. E. 2010, ApJ, 709, 332

Berger, E., Basri, G., Gizis, J. E., Giampapa, M. S., Rutledge, R. E., Liebert, J., Martín, E., Fleming, T. A., Johns-Krull, C. M., Phan-Bao, N., \& Sherry, W. H. 2008, ApJ, 676, 1307

Berger, E., Rutledge, R. E., Phan-Bao, N., Basri, G., Giampapa, M. S., Gizis, J. E., Liebert, J., Martín, E., \& Fleming, T. A. 2009, ApJ, 695, 310

Berger, E., Rutledge, R. E., Reid, I. N., Bildsten, L., Gizis, J. E. Liebert, J., Martín, E., Basri, G., Jayawardhana, R., Brandeker, A., Fleming, T. A., Johns-Krull, C. M., Giampapa, M. S., Hawley, S. L., \& Schmitt, J. H. M. M. 2005, ApJ, 627, 960

Burgasser, A. J., Kirkpatrick, J. D., Reid, I. N., Liebert, J., Gizis, J. E., \& Brown, M. E. 2000, AJ, 120, 473

Burgasser, A. J., \& Putman, M. E. 2005, ApJ, 626, 486

Burgasser, A. J., Sitarski, B. N., Gelino, C. R., Logsdon, S. E., \& Perrin, M. D. 2011, ApJ, 739, 49

Ciliegi, P., Zamorani, G., Hasinger, G., Lehmann, I., Szokoly, G., \& Wilson, G. 2003, A\&A, 398, 901

Condon, J. J., Cotton, W. D., Greisen, E. W., Yin, Q. F., Perley, R. A., Taylor, G. B., \& Broderick, J. J. 1998, AJ, 115, 1693

Dulk, G. A. 1985, ARA\&A, 23, 169

Fleming, T. A., Schmitt, J. H. M. M., \& Giampapa, M. S. 1995, ApJ, 450, 401

Fuhrmeister, B., Schmitt, J. H. M. M., \& Hauschildt, P. H. 2005, A\&A, 439, 1137

Gizis, J. E. 2002, ApJ, 575, 484

Gizis, J. E., Monet, D. G., Reid, I. N., Kirkpatrick, J. D., Liebert, J., \& Williams, R. J. 2000, AJ, 120, 1085

Greisen, E. W. 2003, Information Handling in Astronomy Historical Vistas, 285, 109

Guedel, M., \& Benz, A. O. 1993, ApJ, 405, L63
Hall, P. B. 2002, ApJ, 564, L89

Hallinan, G., Antonova, A., Doyle, J. G., Bourke, S., Lane, C., \& Golden, A. 2008, ApJ, 684, 644

Hallinan, G., Bourke, S., Lane, C., Antonova, A., Zavala, R. T., Brisken, W. F., Boyle, R. P., Vrba, F. J., Doyle, J. G., \& Golden, A. 2007, ApJ, 663, L25

James, D. J., Jardine, M. M., Jeffries, R. D., Randich, S., Collier Cameron, A., \& Ferreira, M. 2000, MNRAS, 318, 1217

Johns-Krull, C. M., \& Valenti, J. A. 1996, ApJ, 459, L95

Kuznetsov, A. A., Doyle, J. G., Yu, S., Hallinan, G., Antonova, A., \& Golden, A. 2012, ApJ, 746, 99

Liebert, J., Kirkpatrick, J. D., Cruz, K. L., Reid, I. N., Burgasser, A., Tinney, C. G., \& Gizis, J. E. 2003, AJ, 125, 343

Machado, M. E., Avrett, E. H., Vernazza, J. E., \& Noyes, R. W. 1980, ApJ, 242, 336

McLean, M., Berger, E., Irwin, J., Forbrich, J., \& Reiners, A. 2011, ApJ, 741, 27

McLean, M., Berger, E., \& Reiners, A. 2012, ApJ, 746, 23

Mohanty, S., Basri, G., Shu, F., Allard, F., \& Chabrier, G. 2002, ApJ, 571, 469

Neupert, W. M. 1968, ApJ, 153, L59

Osten, R. A., Hawley, S. L., Bastian, T. S., \& Reid, I. N. 2006, ApJ, 637, 518

Osten, R. A., Phan-Bao, N., Hawley, S. L., Reid, I. N., \& Ojha, R. 2009, ApJ, 700, 1750

Phan-Bao, N., Osten, R. A., Lim, J., Martín, E. L., \& Ho, P. T. P. 2007, ApJ, 658, 553

Ravi, V., Hallinan, G., Hobbs, G., \& Champion, D. J. 2011, ApJ, $735, \mathrm{~L} 2$

Reiners, A., \& Basri, G. 2010, ApJ, 710, 924

Reiners, A., \& Christensen, U. R. 2010, A\&A, 522, A13+

Route, M., \& Wolszczan, A. 2012, ApJ, 747, L22

Schmidt, S. J., Cruz, K. L., Bongiorno, B. J., Liebert, J., \& Reid, I. N. 2007, AJ, 133, 2258

Schrijver, C. J. 2009, ApJ, 699, L148

Stelzer, B., Micela, G., Flaccomio, E., Neuhäuser, R., \& Jayawardhana, R. 2006, A\&A, 448, 293

Wilson, W. E., Ferris, R. H., Axtens, P., Brown, A., Davis, E., Hampson, G., Leach, M., Roberts, P., Saunders, S., Koribalski, B. S., Caswell, J. L., Lenc, E., Stevens, J., Voronkov, M. A., Wieringa, M. H., Brooks, K., Edwards, P. G., Ekers, R. D., Emonts, B., Hindson, L., Johnston, S., Maddison, S. T., Mahony, E. K., Malu, S. S., Massardi, M., Mao, M. Y., McConnell, D., Norris, R. P., Schnitzeler, D., Subrahmanyan, R., Urquhart, J. S., Thompson, M. A., \& Wark, R. M. 2011, MNRAS, 416, 832

Wu, C. S., \& Lee, L. C. 1979, ApJ, 230, 621

Zarka, P., Queinnec, J., \& Crary, F. J. 2001, Planet. Space Sci., 49, 1137 